\title{
Characteristics of Body Composition and Lifestyle in Chinese University Students with Normal-Weight Obesity: A Cross-Sectional Study
}

\author{
Gusonghan Maitiniyazi ${ }^{1, *}$ \\ Yue Chen ${ }^{1, *}$ \\ Yu-Yu Qiu' \\ Zhen-Xing $X_{i e}{ }^{2}$ \\ Jian-Yun $\mathrm{He}^{\prime}$ \\ Shu-Fang Xia' \\ 'Wuxi School of Medicine, Jiangnan \\ University, Wuxi, Jiangsu, People's \\ Republic of China; ${ }^{2}$ School of Basic \\ Medicine, Henan University, Kaifeng, \\ Henan, People's Republic of China \\ *These authors contributed equally to \\ this work
}

\begin{abstract}
Purpose: Normal weight obesity (NWO), defined as normal body mass index (BMI) and excessive body fat percentage $(\mathrm{BF} \%)$, has been shown to be associated with a significantly higher risk of developing metabolic syndrome, cardiometabolic dysfunction and with higher mortality. However, there is limited literature regarding the potential associations between NWO and lifestyles. This study aimed to investigate the associations of lifestyles with NWO in Chinese university students.
\end{abstract}

Participants and Methods: A total of 279 university students with normal BMI were recruited and divided into NWO and normal weight non-obesity (NWNO) groups by BF\%. Body composition and anthropometrics were measured, and participants were asked to finish the Healthy Lifestyle Scale for University Students (HLSUS) questionnaire.

Results: A total of 26 male (25.5\%) and 71 female (40.1\%) students were identified as NWO. Compared to NWNO students, body weight, BMI, body fat mass, visceral fat area, waist circumference and hip circumference of NWO students were all significantly higher both in male and female students $(P<0.05)$. The body fat mass, $\mathrm{BF} \%$ and visceral fat area were significantly negatively correlated with the total HLSUS, physical exercise behavior, and dietary nutrition behavior scores in NWNO males, NWO and NWNO females $(P<0.05)$. The risk of NWO was lower in those students with higher scores in physical exercise behavior in both males $(\mathrm{OR}=0.298,95 \% \mathrm{CI}=0.121 \sim 0.733)$ and females $(\mathrm{OR}=0.653,95 \% \mathrm{CI}=0.505 \sim 0.843)$, while dietary nutrition behavior $(\mathrm{OR}=0.759,95 \% \mathrm{CI}=0.584 \sim 0.986)$ and stress management behavior $(\mathrm{OR}=0.503,95 \% \mathrm{CI}=0.335 \sim 0.755)$ decreased the risk of NWO only in females.

Conclusion: The incidence of NWO was high among university students, especially in females, which might be related with unhealthy lifestyles. NWO university students should pay attention to lifestyle adjustments, especially physical exercise, dietary nutrition and stress management, for preventing the health risk in NWO.

Keywords: obesity, body fat, physical exercise, dietary nutrition, stress management, public health

\section{Introduction}

Obesity is a severe public health problem that has reached epidemic levels and is developing rapidly. ${ }^{1}$ In China, approximately $46 \%$ of adults and $15 \%$ of children are obese or overweight ${ }^{2,3}$ and the obesity population is increasing dramatically with elevated consumption of high-calorie foods and adoption of a more sedentary lifestyle. ${ }^{4}$ Overweight and obesity have been proven to be both important risk factors for many chronic diseases, such as type 2 diabetes, cardiovascular diseases, musculoskeletal disorders, and some cancers. ${ }^{5,6}$ The recent increasing trend of
Correspondence: Shu-Fang Xia Wuxi School of Medicine, Jiangnan University, Lihu Avenue 1800, Wuxi, 214I22, People's Republic of China $\mathrm{Tel} / \mathrm{Fax}+86-510-8532-8363$

Email xiashufang@jiangnan.edu.cn 
obesity affects young people, especially university students. $^{7}$ Studies have found that first-year university students gained body weight significantly, followed by an ongoing slow but steady increase in weight, ${ }^{8}$ which has attracted full attention from researchers.

As an indirect measure of obesity, body mass index (BMI) is easy and commonly used. However, the sensitivity and specificity of BMI are poor because of the nonlinear relation between it and body fat. Since BMI cannot differentiate between fat and lean tissue, especially in individuals with BMI lower than $30 \mathrm{~kg} / \mathrm{m}^{2}$ but excessive body fat, ${ }^{9,10}$ therefore, researchers suggest that more reliable evaluation indicators should be selected to identify whether an individual is obese. Normal weight obesity (NWO), defined as a combination of normal BMI and high body fat percentage (BF\%) levels, ${ }^{11}$ was at a greater risk of developing non-communicable diseases (NCD) like cardiac metabolic disorders, metabolic syndrome, and cardiovascular diseases. ${ }^{12,13}$ The prevalence of NWO in the Chinese population is relatively high. According to the Chinese NWO criteria, the prevalence in different age groups is $6.71 \% \sim 10.16 \%$ for males and $1.73 \% \sim 6.64 \%$ for females. ${ }^{14}$ Children and adolescents who are NWO may be at increased risk for cardiometabolic morbidity in adulthood. ${ }^{15}$ However, compared to overweight and obesity, it is difficult for university students to identify themselves as NWO based solely on BMI, which causes some NWO students to progressively have metabolic disorders without any intervention. Therefore, identifying the factors that determine the occurrence of NWO will help university students take effective interventions to prevent the NWO progress.

Compared with individuals who are normal weight nonobesity (NWNO) demonstrated by normal BMI and normal $\mathrm{BF} \%$, NWO individuals showed changes in body composition, and increased inflammation and oxidative stress. ${ }^{16}$ Genetics, diet, and physical activity are possible etiological factors for the occurrence of NWO ${ }^{16}$ However, the effective intervention to prevent or treat NWO is still unclear. Unhealthy lifestyles are considered a significant cause of excessive body fat accumulation among university students. ${ }^{17,18}$ It has been reported that NWO, overweight and obese people have a significantly higher incidence of unhealthy lifestyles (such as poor diet, sedentary behavior and lack of physical activity) than people with normal fat., ${ }^{7,19}$ Considering the importance of unhealthy lifestyles on body composition and potential health threats, it is extremely urgent to find the potential link between them in young university students, particularly in NWO students. Therefore, the objective of this study was to differentiate the lifestyles that affect
NWO by investigating the differences and relationships between lifestyles and body composition in NWO and NWNO university students.

\section{Participants and Methods Study Participants}

This cross-sectional study was conducted with 372 university students (232 females and 140 males), including undergraduate and postgraduate students between the ages of 18 to 27 years old $(21.7 \pm 2.1)$ from one university in China from November 2019 to January 2021. This study was approved by the Ethical Committee of Jiangnan University. We recruited participants by distributing leaflets in public areas of the university, such as self-study classrooms, library, canteens, and so on. We also encouraged the participants to forward relevant information about the project on Qzone or WeChat Moments to attract more university students. All participants participated voluntarily. After receiving oral and written information about the research purpose and procedures, they signed an informed consent form, followed by questionnaire surveys, anthropometrics and body composition determination. The inclusion criteria for participants were undergraduates from freshman to senior year and postgraduates. Participants had to be apparently healthy and free from ailments. Exclusion criteria included chronic diseases such as diabetes and endocrine diseases and long-term use of oral medications that affected body weight, such as estrogen or glucocorticoids. In this study, underweight was defined as BMI $<18.5$, overweight was defined as $24 \leqq \mathrm{BMI} \leqq 27.9$, while obesity was defined as BMI $\geqq 28$ based on the criteria of the Working Group on Obesity in China (WGOC), ${ }^{20}$ students with excessive BF\% $(\geqq$ $20 \%$ in male and $\geqq 30 \%$ in female), but normal BMI ( $18.5 \leqq$ BMI $\leqq 23.9)$ were designated as NWO, while students with normal BMI $(18.5 \leqq \mathrm{BMI} \leqq 23.9)$ and normal BF\% $(<20 \%$ in male and $<30 \%$ in female) were defined as NWNO based on the previously published literatures. ${ }^{21,22}$ Figure 1 provides a detailed flow chart of the study participants.

\section{Determination of Sample Size}

The formula for determining the sample size is shown below. Expecting a 95\% confidence interval (CI), the prevalence of NWO was $32 \%$ based on a study by Kim et al. ${ }^{23}$ The required sample size was $\geqq 363$ :

$$
n=\frac{Z_{\alpha / 2}^{2} \times p \times(1-p)}{\delta^{2}}
$$

$n=1.96^{2} \times 0.32 \times(1-0.32) /(0.15 \times 0.32)^{2}=0.8359 / 0.0023=363$ 
Where $\alpha=0.05$, confidence level $=1-\alpha=95 \%, \mathrm{Z}_{\alpha / 2}=$ 1.96, $p$ was the prevalence of NWO and $p=0.32, \delta$ was the permissible error and $\delta=0.15 p=0.048$.

\section{Healthy Lifestyle Questionnaire}

The questionnaire included general characteristics and Wang's Healthy Lifestyle Scale for University Students (HLSUS). ${ }^{24}$ The general characteristics of subjects included sex, age, family monthly income, parents' education level, residence before entering university, and whether the family has only one child. HLSUS contains 38 items with a total of 8 dimensions, including physical exercise behavior, regular life behavior, dietary nutrition behavior, health risk behavior, health responsibility behavior, social support behavior, stress management behavior, and life appreciation behavior. According to the Likert 5 score system, the corresponding score of "never, occasionally, about half, often, always" is 1 to 5 , while the reverse question is 5 to 1 . The Cronbach's $\alpha$ coefficient for the total scale was 0.892 . The HLSUS has good construct

University students $(\mathrm{N}=372)$

(140 males \& 232 females)

\section{Definitions :}

Underweight: BMI $<18.5$

Normal weight: $18.5 \leqq \mathrm{BMI} \leqq 23.9$

Overweight: $24 \leqq \mathrm{BMI} \leqq 27.9$

Obesity: BMI $\geqq 28$

\section{Excluded $(\mathrm{N}=93)$ :}

Underweight: $\mathrm{N}=50$ (10 males \& 40 females)

Overweight: $N=23$ (14 males \& 9 females)

Obesity: $N=20$ ( 14 males $\& 6$ females)

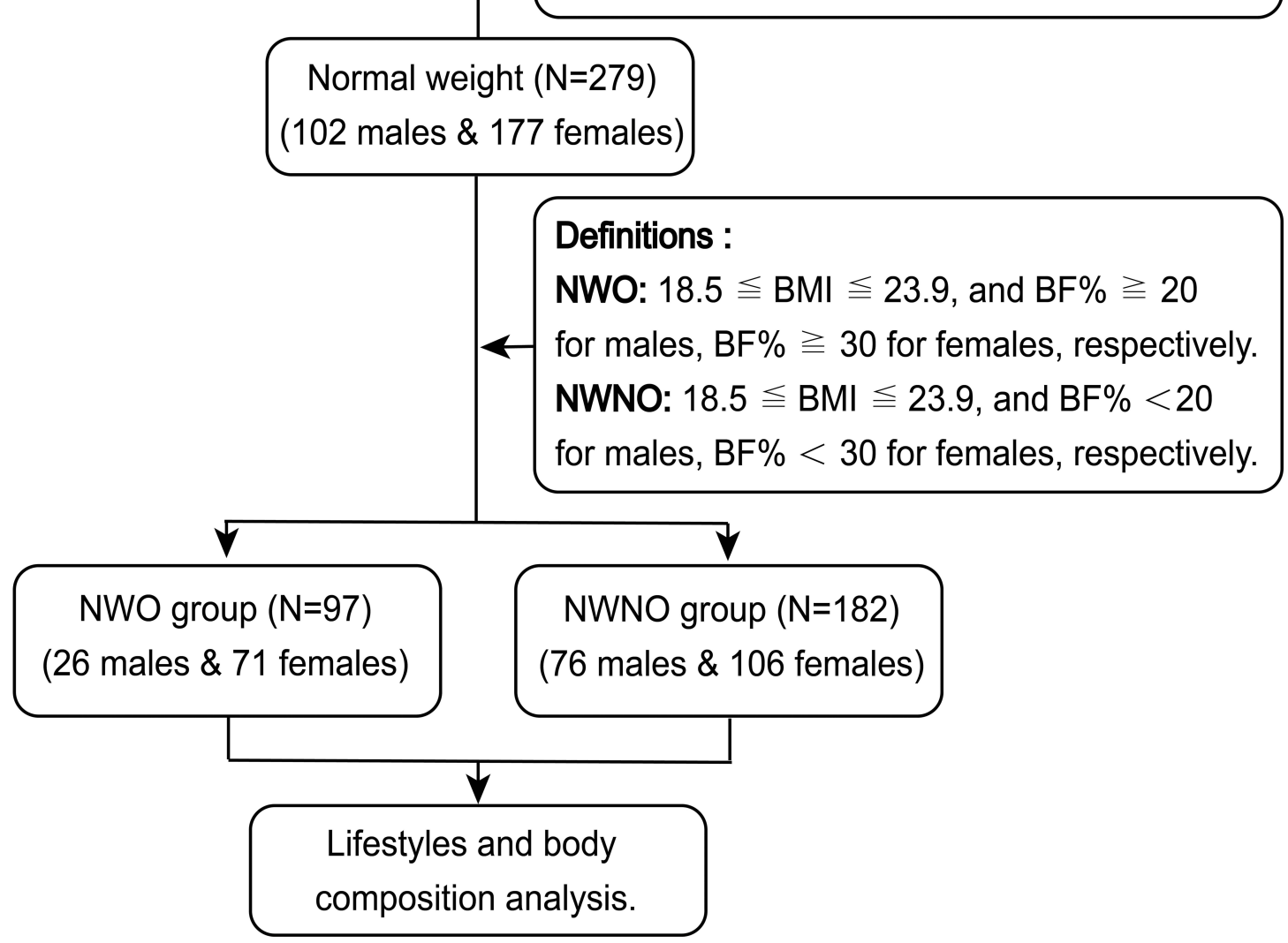

Figure I Flow chart of study participants.

Abbreviations: BMI, body mass index; BF\%, body fat percentage; NWO, normal weight obesity; NWNO, normal weight non-obesity. 
validity and reliability, and it has been widely used in China as an evaluation tool for health counselling in university health centers. ${ }^{24,25}$

\section{Anthropometrics Measurement}

Participants' body height $(\mathrm{cm})$ and weight $(\mathrm{kg})$ were measured to the nearest $0.1 \mathrm{~cm}$ and $0.1 \mathrm{~kg}$, respectively, with participants wearing light clothing without shoes. BMI was calculated by dividing body weight $(\mathrm{kg})$ by height squared $\left(\mathrm{m}^{2}\right)$. Waist circumference (WC) and hip circumference $(\mathrm{HC})$ were determined in duplicate to the nearest $0.5 \mathrm{~cm}$ with a flexible but inelastic measuring tape when the participant was standing relaxed. If the difference between the two measurements exceeded $1 \mathrm{~cm}$, the third measurement would be performed. The mean of the two closest measurements was used for data analysis. Waist was taken at the level of the narrowest part of the torso. If it was difficult to recognize a waist narrowing, the smallest horizontal circumference in the part between the last rib and iliac crest was determined. HC was determined at the maximum circumference of the buttocks posteriorly and the symphysis pubis anteriorly, in a horizontal plane. Waist-to-hip ratio (WHR) was calculated by dividing WC by HC.

\section{Body Composition Assessment}

The body composition of participants was determined by body composition analyzer InBody 770 bioimpedance device (Biospace, Korea). The participants were asked to overnight fasting before the body composition assessment. Two hours before the test, the participants did not go on exercise vigorously. They wore light clothes, removed metal jewelry and watches, took off their shoes and socks, drooped their limbs, and separated upper limbs from their torso by about 15 degrees. After inputting the participants' personal information, body fat mass, $\mathrm{BF} \%$, total body water, soft lean mass, fat-free mass, skeletal muscle mass, visceral fat area, protein content and mineral content were automatically determined.

\section{Statistical Analyses}

Data analyses were performed using SPSS 22.0 (SPSS Inc., Chicago, IL, USA). Data were expressed as mean $\pm \mathrm{SD}$, and categorical variables were presented as frequencies (N) and percentages (\%). Chi-square test was used to analyze the differences between categorical variables. Independent sample $t$-test was used to analyze the differences in lifestyles and body composition between NWO and NWNO university students of different sex. Pearson correlation analysis was used to examine the correlations among lifestyles and body composition. Finally, in order to investigate the relationships between whether university student was NWO and the lifestyles, a binary logistic regression analysis was conducted, NWO was taken as dependent variables, the lifestyle behaviors with statistical significance in univariate analysis were taken as independent variables, and the multivariate logistic regression analysis was used. Univariate and multivariate logistic regression analyses were both performed with adjustment for variables age, family monthly income, father's educational level, mother's educational level, residence before entering university, and whether the only child. The Hosmer-Lemeshow test was used to evaluate the goodness of fit between the data and the binary logistic regression. The model fitting accuracy was high, and there was no multicollinearity. A value of $P<0.05$ was considered statistically significant.

\section{Results}

\section{Demographic Characteristics of the Study Participants}

Out of the 372 participants (140 males and 232 females), $10(7.1 \%)$ males and $40(17.2 \%)$ of females were underweight, $14(10.0 \%)$ males and $9(3.9 \%)$ females were overweight, and $14(10.0 \%)$ males and $6(2.6 \%)$ females were obesity, respectively. Fifty-six (15.1\%) participants were freshmen, 91 (24.4\%) participants were sophomores, $71(19.1 \%)$ participants were juniors, 80 (21.5\%) participants were seniors, and 74 (19.9\%) participants were postgraduate students. A total of 102 (72.9\%) males and 177 $(76.3 \%)$ females were classified as normal weight and participated in the study, of which $26(25.5 \%)$ males and $71(40.1 \%)$ females were classified as NWO, while 76 (74.5\%) males and 106 (59.9\%) females were NWNO. The demographic characteristics of the 279 subjects with normal BMI are shown in Supplementary Table 1. No statistically significant differences were detected in age, father's educational level, mother's educational level, residence before entering university and whether the only child between the NWO and NWNO in both sexes $(P>$ $0.05)$. There was a statistical difference in family monthly income between NWO and NWNO in females $(P<0.01)$, but not in males. 


\section{Anthropometric Indicators and Body Composition of University Students}

Anthropometric indicators and body composition of university students are demonstrated in Table 1. Compared to NWNO students, body weight, BMI, body fat mass, BF\%, visceral fat area, waist circumference, and hip circumference of NWO students were all significantly higher both in male and female students $(P<0.05)$. In addition, NWNO female students demonstrated significantly higher fat-free mass $(P=0.028)$ and skeletal muscle mass $(P=0.024)$ than NWO female students.

\section{Healthy Lifestyles of University Students}

The scores of the HLSUS are shown in Table 2. Total HLSUS scores, physical exercise behavior scores, regular life behavior scores, dietary nutrition behavior scores and stress management behavior scores of NWNO students were all remarkably higher than those of NWO students in both males and females $(P<0.01)$. However, significant differences on life appreciation behavior scores between NWO and NWNO groups were only found in females $(P<0.01)$.

\section{Correlations Among Body Composition and Lifestyles of University Students}

The body fat mass, BF\% and visceral fat area were significantly negatively correlated with the total HLSUS, physical exercise behavior, and dietary nutrition behavior in NWNO males, NWO and NWNO females, and also negatively correlated with regular life behavior and stress management behavior in NWNO male students $(P<0.05)$ (Table 3). In NWO females, the $\mathrm{BF} \%$ was negatively correlated with stress management behavior and life appreciation behavior, while the visceral fat area was negatively correlated with life appreciation behavior, respectively $(P<0.05)$. BMI was negatively correlated with NWO male and NWNO female students' dietary nutrition behavior, and NWO female students' total HLSUS and physical exercise behavior $(P<0.05)$.

\section{Binary Logistic Regression Analyses of NWO University Students}

Univariate logistic regression analyses between NWO and lifestyles revealed that physical exercise behavior, regular life behavior, dietary nutrition behavior and stress management behavior significantly decreased the risk of NWO in both male and female students, while the life appreciation behavior only decreased the risk of NWO in female students $(P<0.05)$ (Supplementary Table 2). Multivariate logistic regression analysis (Table 4) further revealed that physical exercise behavior was the independent protective factor for NWO in males (OR $=0.298,95 \% \mathrm{CI}=0.121 \sim 0.733$ ), while physical exercise behavior $(\mathrm{OR}=0.653,95 \% \mathrm{CI}=0.505 \sim 0.843)$, dietary nutrition behavior $(\mathrm{OR}=0.759,95 \% \mathrm{CI}=0.584 \sim 0.986)$ and stress

Table I Anthropometric Indicators and Body Composition of Participants Stratified by Sex and Body Fat Percentage

\begin{tabular}{|c|c|c|c|c|}
\hline & \multicolumn{2}{|c|}{ Male $(n=102)$} & \multicolumn{2}{|c|}{ Female $(n=177)$} \\
\hline & NWO $(n=26)$ & NWNO $(n=76)$ & NWO (n = 7I) & NWNO $(n=106)$ \\
\hline Body weight (kg) & $68.8 \pm 6.8$ & $63.5 \pm 8.6^{* *}$ & $55.7 \pm 5.4$ & $52.6 \pm 4.6 * *$ \\
\hline Body height $(\mathrm{cm})$ & $171.7 \pm 6.9$ & $175.0 \pm 7.1$ & $162.9 \pm 5.7$ & $161.6 \pm 4.7$ \\
\hline BMI $\left(\mathrm{kg} / \mathrm{m}^{2}\right)$ & $23.3 \pm 0.5$ & $20.6 \pm 1.6 * *$ & $21.0 \pm 1.6$ & $20.1 \pm 1.2^{* *}$ \\
\hline Body fat mass (kg) & $16.5 \pm 3.4$ & $9.2 \pm 2.5 * *$ & $18.2 \pm 2.6$ & $13.9 \pm 2.0 * *$ \\
\hline $\mathrm{BF}(\%)$ & $23.9 \pm 3.6$ & $14.4 \pm 2.7^{* *}$ & $32.7 \pm 2.7$ & $26.4 \pm 2.5^{* *}$ \\
\hline Total body water (L) & $38.4 \pm 3.8$ & $39.7 \pm 5.1$ & $27.5 \pm 2.7$ & $28.2 \pm 2.5$ \\
\hline Soft lean mass $(\mathrm{kg})$ & $49.4 \pm 4.8$ & $51.2 \pm 6.6$ & $35.1 \pm 3.4$ & $36.1 \pm 3.6$ \\
\hline Fat-free mass $(\mathrm{kg})$ & $52.4 \pm 5.2$ & $54.2 \pm 7.0$ & $37.5 \pm 3.7$ & $38.6 \pm 3.4 *$ \\
\hline Skeletal muscle mass $(\mathrm{kg})$ & $29.2 \pm 2.9$ & $30.5 \pm 4.2$ & $20.0 \pm 1.96$ & $20.7 \pm 2.0 *$ \\
\hline Bone mineral content $(\mathrm{kg})$ & $2.96 \pm 0.36$ & $3.06 \pm 0.46$ & $2.30 \pm 0.22$ & $2.37 \pm 0.24$ \\
\hline Visceral fat area $\left(\mathrm{cm}^{2}\right)$ & $67.8 \pm 17.1$ & $33.6 \pm 12.2^{* *}$ & $80.9 \pm 18.2$ & $55.2 \pm 8.4^{* *}$ \\
\hline Waist circumference $(\mathrm{cm})$ & $80.7 \pm 7.1$ & $74.4 \pm 6.9 * *$ & $69.3 \pm 4.3$ & $67.9 \pm 4.8^{*}$ \\
\hline Hip circumference $(\mathrm{cm})$ & $98.9 \pm 4.9$ & $92.0 \pm 6.4^{* *}$ & $94.1 \pm 4.3$ & $90.9 \pm 4.2^{* *}$ \\
\hline WHR & $0.81 \pm 0.05$ & $0.81 \pm 0.04$ & $0.74 \pm 0.04$ & $0.75 \pm 0.04$ \\
\hline
\end{tabular}

Notes: $* \mathrm{P}<0.05$ (NWO vs NWNO); **P < 0.01 (NWO vs NWNO).

Abbreviations: NWO, normal weight obesity; NWNO, normal weight non-obesity; BMI, body mass index; BF\%, body fat percentage; WHR, waist-to-hip ratio. 
Table 2 Healthy Lifestyles Scale of University Students Stratified by Sex and Body Fat Percentage

\begin{tabular}{|c|c|c|c|c|c|}
\hline \multirow[t]{2}{*}{ HLSUS and Dimension } & \multirow{2}{*}{$\begin{array}{l}\text { Range of Obtainable } \\
\text { Scores (Min and Max.) }\end{array}$} & \multicolumn{2}{|c|}{ Male $(n=102)$} & \multicolumn{2}{|c|}{ Female $(n=177)$} \\
\hline & & NWO (n = 26) & NWNO (n = 76) & NWO (n = 7I) & NWNO $(n=106)$ \\
\hline Total HLSUS (38 items) & $38-190$ & $143.3 \pm 10.6$ & $154.0 \pm 11.5^{* *}$ & $139.4 \pm 9.5$ & $\mid 47.0 \pm 8.1^{* *}$ \\
\hline Physical exercise behavior & $4-20$ & $12.5 \pm 2.0$ & $15.6 \pm 2.3^{* *}$ & $11.4 \pm 1.5$ & $13.1 \pm 2.6^{* *}$ \\
\hline Regular life behavior & $4-20$ & $13.1 \pm 1.4$ & $15.1 \pm 2.1 * *$ & $12.8 \pm 2.4$ & $14.3 \pm 2.2^{* *}$ \\
\hline Dietary nutrition behavior & $4-20$ & $14.4 \pm 2.6$ & $16.5 \pm 1.9 * *$ & $13.2 \pm 1.6$ & $14.5 \pm 2.4^{* *}$ \\
\hline Health risk behavior & $4-20$ & $12.4 \pm 2.1$ & $13.3 \pm 1.4$ & $13.5 \pm 1.4$ & $13.4 \pm 1.3$ \\
\hline Health responsibility behavior & $6-30$ & $26.1 \pm 2.2$ & $26.0 \pm 2.2$ & $26.2 \pm 2.7$ & $26.2 \pm 2.1$ \\
\hline Social support behavior & $6-30$ & $24.6 \pm 1.8$ & $25.4 \pm 2.0$ & $24.6 \pm 2.4$ & $25.2 \pm 2.1$ \\
\hline Stress management behavior & $5-25$ & $19.8 \pm 1.7$ & $21.0 \pm 1.7^{* *}$ & $18.7 \pm 1.8$ & $20.0 \pm 1.9 * *$ \\
\hline Life appreciation behavior & $5-25$ & $20.4 \pm 2.3$ & $21.2 \pm 2.4$ & $18.9 \pm 2.4$ & $20.4 \pm 1.9 * *$ \\
\hline
\end{tabular}

Note: **P $<0.01$ (NWO vs NWNO).

Abbreviations: NWO, normal weight obesity; NWNO, normal weight non-obesity; HLSUS, healthy lifestyles scale of university students.

management behavior $(\mathrm{OR}=0.503,95 \% \mathrm{CI}=0.335 \sim 0.755)$ were the protective factors in females.

\section{Discussion}

In this study, we studied the lifestyles that might determine NWO in Chinese university students by investigating the differences and associations on lifestyles and body composition between NWO and NWNO individuals. The primary findings were that the incidence of NWO was high among university students, especially in females. Compared with NWO students, NWNO students showed healthier lifestyles, especially in terms of physical exercise and dietary nutrition. Furthermore, physical exercise was an independent protective factor for NWO in both sexes, while dietary nutrition and stress management were also protective factors for NWO only in females.

Typically, obesity has been classified based on BMI, because of its simplicity and validation in a variety of epidemiologic studies. ${ }^{26}$ However, NWO is more likely to be ignored by people because it cannot be easily recognized by BMI. Researchers found that half of the people with excessive $\mathrm{BF} \%$ were failed to identify only based on BMI. $^{27}$ The reason might be that BMI cannot distinguish between lean and fat tissue, especially in subjects with normal BMI but excessive body fat. ${ }^{28}$ Studies have confirmed that NWO has become a critical factor affecting the health of university students and is associated with metabolic disorders, cardiovascular disease, as well as an increase in all-cause mortality. ${ }^{12,29}$ Therefore, timely detection and intervention of NWO is the key to prevent university students from obesity and other metabolic diseases. Currently, the cutoffs of BF\% used to identify NWO have varied, depending on the study population, sex and ethnicity of the studied populations, ${ }^{29}$ and no clear criteria have been set. The cut-off value for NWO people from the Asia-Pacific region is $\geqq 33.4 \%$ in women and $\geqq 20.6 \%$ in men. ${ }^{11}$ We referred to the definition of $\mathrm{NWO}^{11}$ and previous Chinese researchers' cut-off value on NWO university students based on $\mathrm{BF} \%$ ( $\geqq 20 \%$ for men and $\geqq 30 \%$ for women), ${ }^{21,22}$ and found that $25.5 \%$ of male students and $40.1 \%$ of female students were classified as NWO in our research, which was higher than the incidence of NWO in both sexes investigated by Zhang et al. ${ }^{21}$ However, we both confirmed that female university students were more susceptible to become NWO. Additionally, NWNO students also had lower body weight, body fat mass, visceral fat area, waist circumference and hip circumference than NWO students in both sexes, indicating that NWNO university students had a healthier body composition.

Healthy lifestyles are directly beneficial to the study and life of university students, and they also play critical roles in the formation and construction of a healthy society as a whole. ${ }^{30}$ Since the prevalence of NWO in university students was relatively high, factors related with body composition, such as body weight, BMI, body fat mass, fat-free mass, visceral fat area, waist and hip circumference, and WHR needed to be identified, in order to keep prevention programs more effective. ${ }^{31}$ It is well known that unhealthy lifestyles, such as sedentary behaviors, low physical exercise levels, and unreasonable dietary behaviors, were essential causes of body fat accumulation in the general public as well as in children and adolescents. $^{32,33}$ An active lifestyle is significantly correlated to improvements on body composition parameters. ${ }^{34}$ In order to evaluate the lifestyles of NWO and NWNO 
Table 3 The Correlations Between Body Composition and Lifestyle Scores

\begin{tabular}{|c|c|c|c|c|c|c|}
\hline & Total HLSUS & $\begin{array}{l}\text { Physical } \\
\text { Exercise } \\
\text { Behavior }\end{array}$ & $\begin{array}{l}\text { Dietary } \\
\text { Nutrition } \\
\text { Behavior }\end{array}$ & $\begin{array}{l}\text { Regular } \\
\text { Life } \\
\text { Behavior }\end{array}$ & $\begin{array}{c}\text { Stress } \\
\text { Management } \\
\text { Behavior }\end{array}$ & $\begin{array}{c}\text { Life } \\
\text { Appreciation } \\
\text { Behavior }\end{array}$ \\
\hline \multicolumn{7}{|l|}{ NWO male $(n=26)$} \\
\hline Body weight & -0.318 & -0.184 & -0.355 & -0.158 & -0.320 & - \\
\hline BMI & -0.058 & -0.233 & $-0.402^{*}$ & -0.174 & -0.027 & - \\
\hline Body fat mass & -0.255 & -0.174 & $-0.432 *$ & -0.308 & -0.228 & - \\
\hline $\mathrm{BF} \%$ & -0.148 & -0.114 & -0.352 & -0.313 & -0.127 & - \\
\hline Visceral fat area & -0.163 & -0.069 & -0.344 & -0.233 & -0.233 & - \\
\hline Waist circumference & -0.061 & -0.082 & -0.097 & -0.185 & -0.156 & - \\
\hline Hip circumference & 0.084 & -0.057 & -0.137 & -0.039 & -0.094 & - \\
\hline \multicolumn{7}{|l|}{ NWNO male $(n=76)$} \\
\hline Body weight & -0.069 & -0.137 & -0.144 & -0.160 & -0.108 & - \\
\hline BMI & -0.117 & -0.156 & -0.155 & -0.092 & -0.165 & - \\
\hline Body fat mass & $-0.367^{* *}$ & $-0.374 * *$ & $-0.343^{* *}$ & $-0.288^{*}$ & $-0.332^{* *}$ & - \\
\hline BF\% & $-0.474 * *$ & $-0.4 I I * *$ & $-0.376 * *$ & $-0.287^{*}$ & $-0.409 * *$ & - \\
\hline Visceral fat area & $-0.384 * *$ & $-0.410 * *$ & $-0.384 * *$ & $-0.351 * *$ & $-0.309 * *$ & - \\
\hline Waist circumference & -0.131 & -0.105 & -0.114 & $-0.14 \mid$ & -0.089 & - \\
\hline Hip circumference & -0.056 & -0.078 & -0.095 & -0.129 & -0.078 & - \\
\hline \multicolumn{7}{|l|}{ NWO female $(n=7 I)$} \\
\hline Body weight & -0.105 & -0.098 & -0.048 & -0.069 & -0.100 & -0.021 \\
\hline BMI & $-0.245^{*}$ & $-0.26 I^{*}$ & -0.220 & -0.052 & -0.157 & -0.020 \\
\hline Body fat mass & $-0.310 * *$ & $-0.338 * *$ & $-0.252^{*}$ & -0.132 & -0.215 & -0.206 \\
\hline $\mathrm{BF} \%$ & $-0.402 * *$ & $-0.462 * *$ & $-0.374 * *$ & -0.149 & $-0.242 *$ & $-0.316 * *$ \\
\hline Fat-free mass & 0.078 & 0.112 & 0.114 & -0.006 & 0.013 & 0.129 \\
\hline Skeletal muscle mass & 0.112 & 0.137 & 0.134 & 0.035 & 0.033 & 0.137 \\
\hline Visceral fat area & $-0.347 * *$ & $-0.409 * *$ & $-0.324 * *$ & -0.176 & -0.203 & $-0.247^{*}$ \\
\hline Waist circumference & -0.176 & -0.088 & -0.016 & -0.102 & -0.031 & 0.117 \\
\hline Hip circumference & -0.091 & -0.163 & 0.060 & 0.018 & -0.153 & -0.055 \\
\hline \multicolumn{7}{|l|}{ NWNO female $(n=106)$} \\
\hline Body weight & -0.167 & -0.110 & $-0.30 I^{* *}$ & 0.021 & 0.182 & 0.005 \\
\hline BMI & -0.073 & -0.105 & $-0.260 *$ & 0.073 & 0.245 & 0.029 \\
\hline Body fat mass & $-0.370 * *$ & $-0.417^{* *}$ & $-0.475^{* *}$ & -0.189 & 0.129 & -0.047 \\
\hline BF\% & $-0.355 * *$ & $-0.502 * *$ & $-0.400 * *$ & $-0.27 I^{*}$ & 0.032 & -0.064 \\
\hline Fat-free mass & -0.004 & -0.099 & -0.126 & 0.149 & 0.163 & 0.030 \\
\hline Skeletal muscle mass & -0.018 & -0.093 & -0.129 & 0.136 & 0.166 & 0.028 \\
\hline Visceral fat area & $-0.314 * *$ & $-0.365^{* *}$ & $-0.408 * *$ & -0.167 & 0.103 & -0.040 \\
\hline Waist circumference & -0.129 & -0.239 & -0.228 & -0.195 & 0.242 & 0.204 \\
\hline Hip circumference & -0.209 & -0.234 & $-0.328 * *$ & -0.010 & 0.145 & 0.051 \\
\hline
\end{tabular}

Notes: $* \mathrm{P}<0.05$, $* * \mathrm{P}<0.01$.

Abbreviations: NWO, normal weight obesity; NWNO, normal weight non-obesity; BMI, body mass index; BF\%, body fat percentage; HLSUS, healthy lifestyles scale of university students.

university students, the HLSUS questionnaire was used. NWNO students demonstrated higher average total HLSUS scores than NWO students, especially in physical exercise, dietary nutrition and regular life behavior scores, implying that the lifestyles of NWO university students were not healthy. Additionally, total HLSUS, physical exercise behavior, and dietary nutrition behavior also significantly negatively correlated with $\mathrm{BF} \%$, indicating that these lifestyle factors might be important in determining whether a university student was NWO or NWNO, which was in accordance with the previous study. ${ }^{35}$

Physical exercise behavior was significantly negatively correlated with body fat mass, $\mathrm{BF} \%$ and visceral fat area in both NWNO males and females, but only in NWO females. In a study, physical activity has been shown to be inversely related with BMI, waist circumference and 
Table 4 Multivariate Logistic Regression Analysis of University Students' Lifestyles and NWO by Sex

\begin{tabular}{|l|l|l|l|}
\hline & OR & $\mathbf{9 5 \%} \mathbf{~ C l}$ & P-value \\
\hline Male & & & \\
Physical exercise behavior & 0.298 & $(0.121,0.733)$ & 0.009 \\
Regular life behavior & 0.600 & $(0.324,1.108)$ & 0.127 \\
Dietary nutrition behavior & 1.661 & $(0.863,3.198)$ & 0.208 \\
Stress management behavior & 0.570 & $(0.305,1.065)$ & 0.130 \\
\hline Female & & & \\
Physical exercise behavior & 0.653 & $(0.505,0.843)$ & 0.001 \\
Regular life behavior & 0.878 & $(0.699,1.103)$ & 0.263 \\
Dietary nutrition behavior & 0.759 & $(0.584,0.986)$ & 0.039 \\
Stress management behavior & 0.503 & $(0.335,0.755)$ & 0.001 \\
Life appreciation behavior & 1.046 & $(0.784,1.397)$ & 0.759 \\
\hline
\end{tabular}

Notes: The multivariate logistic regression models were adjusted for age, family monthly income, father's educational level, mother's educational level, residence before entering university, and whether the only child.

Abbreviation: NWO, normal weight obesity.

fat-mass percentage and positively related with fat-free mass percentage. ${ }^{34}$ However, we failed to find the significant relations between physical activity, BMI, and waist circumference. Although the sample sizes were similar, we did not directly analyze the correlations by sex, but further divided the males and females into NWO and NWNO groups and then analyzed the correlations, respectively, which might lead to the differences in the two studies. NWO had also been demonstrated to be associated with poor physical fitness, and the relationship was partially regulated by lower skeletal muscle mass. ${ }^{21}$ We found the difference on skeletal muscle mass between NWO and NWNO females, but did not find the significance in males, possibly because of the small sample size in NWO males, and further analysis failed to reveal the correlation between skeletal muscle mass and physical exercise behavior, which might be attributed to the different ways to evaluate physical activity.

Dietary factors also exert important roles in body composition. The intakes of energy-yielding nutrients were not associated with NWO. Instead, some healthy dietary factors (low intakes of meat and soft drinks) as well as unhealthy factors (low intakes of root vegetables, cereals and fish and high intake of confectionery) were related to NWO. ${ }^{36}$ Despite the fact that NWO male university students appeared lean, they have been reported to consume lower amounts of dietary antioxidants and demonstrated the same low body antioxidant status as obese individuals because of the unhealthy diet. ${ }^{37}$ The above findings suggest that a reasonable diet is critical for maintaining healthy body composition. We found a significant inverse correlation between dietary nutrition behavior and body fat mass and $\mathrm{BF} \%$, respectively, in NWO and NWNO females, as well as NWNO males. We also found that dietary nutrition behavior was an independent protective factor for NWO in females, further emphasizing the positive role of nutrition in NWO prevention and treatment. However, it had been reported that self-regulation of eating and physical activity was lower in obese female college students as compared to their normal weight counterparts. ${ }^{38}$ Physical activity and dietary behaviors both play a substantial action in subjects becoming overweight and developing obesity, and they were the most modifiable factors, ${ }^{39}$ we believe that if NWO students want to become NWNO, healthy lifestyle adjustments, particularly physical exercise and dietary nutrition, should be focused.

Obesity is a known risk factor for many chronic conditions, as well as mental health disorders. ${ }^{40}$ Obesity will progress from childhood to adulthood without effective intervention, followed by a series of expanding related physical and mental health problems. ${ }^{41} \mathrm{We}$ found that stress management behavior and life appreciation behavior scores of NWNO female students were significantly higher than those of the NWO, and these two indicators remarkably negatively correlated with $\mathrm{BF} \%$. Regression analysis revealed that the risk of NWO was lower in females with a higher score in stress management behavior, which was consistent with the previous researches that stress had been shown as a key risk factor affecting obesity, ${ }^{42}$ and psychological stress in the family led to childhood obesity. ${ }^{43}$ Stress can lead to obesity through inappropriate eating behavior, ${ }^{44}$ while relaxation can reduce emotional eating and improve cognitive restraint, thereby reducing weight. $^{45}$ In addition, people who are stressed are also the most difficult to lose weight. ${ }^{46}$ Stress management plays an important role in reducing weight, improving body composition and preventing various comorbidities. $^{47}$ Therefore, it is important to try to help university students identify the source of stress and maintain a good mental state.

There are some additional limitations on this research that should be considered. First, the HLSUS questionnaire content is limited, which cannot summarize all the situations involved in the lifestyles, leading to incomplete surveys. Second, because of the cross-sectional design, we cannot exclude the possibility of reverse causality. Finally, we conducted a study on a small sample $(\mathrm{N}=$ 
279) of university students. Only 26 male students were classified as NWO, which might affect the accuracy of the results. Therefore, large-scale cross-sectional studies or longitudinal studies are needed to determine the factors of NWO in the future.

\section{Conclusion}

This study showed that $25.5 \%$ of male and $40.1 \%$ of female university students with normal BMI were classified as NWO. NWO university students had lower HLSUS, physical exercise, dietary nutrition, regular life and stress management scores than their NWNO peers, which might directly lead to significantly higher BMI, body fat mass and percentage, visceral fat area. Among the lifestyles, physical exercise was the independent protective factor for NWO in both sexes, while dietary nutrition and stress management were also protective factors for NWO in female students. These findings indicated that much attention should be paid for the lifestyle adjustments, especially physical exercise, dietary nutrition and stress management behavior, for preventing the health risk in NWO university students.

\section{Ethics Approval and Consent to Participate}

This study was conducted in accordance with the Declaration of Helsinki. This study was approved by the Ethical Committee of Jiangnan University. Written informed consent was obtained from all subjects involved in the study.

\section{Consent for Publication}

Consent for publication was obtained from every individual whose data are included in this manuscript.

\section{Acknowledgments}

The authors are grateful to Yu-Qiu Ge and Li-Mei Chen for the statistical analysis provided and Ting-Ting Cui, Qing-Yu Liu, Zi-Yu Hu, and Lan Cheng for their assistance in collecting data.

\section{Funding}

This research was funded by the National Natural Science Foundation of China, grant number 81703222; China Postdoctoral Science Foundation, grantnumber 2017M620191; Postdoctoral Foundation of Jiangsu Province, grant number 1701099B.

\section{Disclosure}

The authors report no conflicts of interest in this work.

\section{References}

1. González-Muniesa P, Mártinez-González MA, Hu FB, et al. Obesity. Nat Rev Dis Primers. 2017;3:17034. doi:10.1038/ nrdp. 2017.34

2. Wang Y, Xue H, Sun M, Zhu X, Zhao L, Yang Y. Prevention and control of obesity in China. Lancet Glob Health. 2019;7 (9):1166-1167. doi:10.1016/s2214-109x(19)30276-1

3. Wang Y, Wang L, Qu W. New national data show alarming increase in obesity and noncommunicable chronic diseases in China. Eur J Clin Nutr. 2017;71(1):149-150. doi:10.1038/ejen.2016.171

4. Popkin BM, Adair LS, Ng SW. Global nutrition transition and the pandemic of obesity in developing countries. Nutr Rev. 2012;70 (1):3-21. doi:10.1111/j.1753-4887.2011.00456.x

5. Liu P, Ye Z, Lu J, et al. A comparison of bone mineral densities and body composition between Southeast Asia college students and Chinese college students. Medicine. 2016;95(37):4724-4728. doi:10.1097/MD.0000000000004724

6. Jiang Y, Wang J, Wu S, et al. Association between take-out food consumption and obesity among Chinese university students: a Cross-Sectional Study. Int J Environ Res Public Health. 2019;16 (6):1071-1083. doi:10.3390/ijerph16061071

7. Stefan L, Cule M, Milinovic I, Juranko D, Sporis G. The relationship between lifestyle factors and body composition in young adults. Int J Environ Res Public Health. 2017;14(8):893-905. doi:10.3390/ijerph14080893

8. Vella-Zarb RA, Elgar FJ. The 'freshman 5': a meta-analysis of weight gain in the freshman year of college. $J$ Am Coll Health. 2009;58 (2):161-166. doi:10.1080/07448480903221392

9. Okorodudu DO, Jumean MF, Montori VM, et al. Diagnostic performance of body mass index to identify obesity as defined by body adiposity: a systematic review and meta-analysis. Int J Obes. 2010;34 (5):791-799. doi:10.1038/ijo.2010.5

10. Romero-Corral A, Somers VK, Sierra-Johnson J, et al. Accuracy of body mass index in diagnosing obesity in the adult general population. Int J Obes. 2008;32(6):959-966. doi:10.1038/ijo.2008.11

11. Franco LP, Morais CC, Cominetti C. Normal-weight obesity syndrome: diagnosis, prevalence, and clinical implications. Nutr Rev. 2016;74(9):558-570. doi:10.1093/nutrit/nuw019

12. Jean N, Somers VK, Sochor O, Medina-Inojosa J, Llano EM, LopezJimenez F. Normal-weight obesity: implications for cardiovascular health. Curr Atheroscler Rep. 2014;16(12):464-472. doi:10.1007/ s11883-014-0464-7

13. Romero-Corral A, Somers V, Sierra-Johnson J, et al. Normal weight obesity: a risk factor for cardiometabolic dysregulation and cardiovascular mortality. Eur Heart J. 2010;31(6):737-746. doi:10.1093/ eurheartj/ehp487

14. Jia A, Xu S, Xing Y, et al. Prevalence and cardiometabolic risks of normal weight obesity in Chinese population: a nationwide study. Nutr Metab Cardiovasc Dis. 2018;28(10):1045-1053. doi:10.1016/j. numecd.2018.06.015

15. Wiklund P, Törmäkangas T, Shi Y, et al. Normal-weight obesity and cardiometabolic risk: a 7-year longitudinal study in girls from prepuberty to early adulthood. Obesity. 2017;25(6):1077-1082. doi:10.1002/oby.21838

16. Wijayatunga NN, Dhurandhar EJ. Normal weight obesity and unaddressed cardiometabolic health risk-a narrative review. Int $J$ Obes. 2021. doi:10.1038/s41366-021-00858-7

17. Brunt AR, Rhee YS. Obesity and lifestyle in U.S. college students related to living arrangements. Appetite. 2008;51(3):615-621. doi:10.1016/j.appet.2008.04.019 
18. Asghar A, Masood Shah A, Ali Hussain A, Tahir A, Asghar H. Frequency of pre-obesity and obesity in medical students of Karachi and the predisposing lifestyle habits. Cureus. 2019;11 (1):3948-3956. doi:10.7759/cureus.3948

19. Mushtaq M, Gull S, Mushtaq K, Shahid U, Shad M, Akram J. Dietary behaviors, physical activity and sedentary lifestyle associated with overweight and obesity, and their socio-demographic correlates, among Pakistani primary school children. Int J Behav Nutrition Phy. 2011;8:130-143. doi:10.1186/1479-5868-8-130

20. Chen C, Lu FC. The guidelines for prevention and control of overweight and obesity in Chinese adults. Biomed Environ Sci. 2004;17: Suppl:1-36.

21. Zhang M, Schumann M, Huang T, Tormakangas T, Cheng S. Normal weight obesity and physical fitness in Chinese university students: an overlooked association. BMC Public Health. 2018;18(1):1334-1344. doi:10.1186/s12889-018-6238-3

22. Tian Q, Wang H, Kaudimba KK, et al. Characteristics of physical fitness and cardiometabolic risk in Chinese university students with normal-weight obesity: a Cross-Sectional Study. Diabetes Metab Syndr Obes. 2020;13:4157-4167. doi:10.2147/dmso.S280350

23. Kim MK, Han K, Kwon HS, et al. Normal weight obesity in Korean adults. Clin Endocrinol (Oxf). 2014;80(2):214-220. doi:10.1111/ cen. 12162

24. Wang D, Xing XH, Wu XB. Healthy lifestyles of university students in China and influential factors. Scientific World J. 2013;2013:412950. doi:10.1155/2013/412950

25. Wang D, Xing XH, Wu XB. The Healthy Lifestyle Scale for University Students: development and psychometric testing. Aust J Prim Health. 2012;18(4):339-345. doi:10.1071/PY11107

26. World Health Organization. Obesity: preventing and managing the global epidemic. Report of a WHO consultation. World Health Organ Tech Rep Ser. 2000;894:1-253.

27. Javed A, Jumean M, Murad MH, et al. Diagnostic performance of body mass index to identify obesity as defined by body adiposity in children and adolescents: a systematic review and meta-analysis. Pediatr Obes. 2015;10(3):234-244. doi:10.1111/ijpo.242

28. Flegal KM, Carroll MD, Kit BK, Ogden CL. Prevalence of obesity and trends in the distribution of body mass index among US adults, 1999-2010. JAMA. 2012;307(5):491-497. doi:10.1001/jama.2012.39

29. Kapoor N, Furler J, Paul TV, Thomas N, Oldenburg B. Normal weight obesity: an underrecognized problem in individuals of south asian descent. Clin Ther. 2019;41(8):1638-1642. doi:10.1016/j. clinthera.2019.05.016

30. Assaf I, Brieteh F, Tfaily M, El-Baida M, Kadry S, Balusamy B. Students university healthy lifestyle practice: quantitative analysis. Health Inf Sci Syst. 2019;7(1):7-19. doi:10.1007/s13755-019-0068-2

31. Ferrari GLM, Matsudo V, Katzmarzyk PT, Fisberg M. Prevalence and factors associated with body mass index in children aged 9-11 years. J Pediatr (Rio J). 2017;93(6):601-609. doi:10.1016/j. jped.2016.12.007

32. Santaliestra-Pasias AM, Mouratidou T, Reisch L, et al. Clustering of lifestyle behaviours and relation to body composition in European children. The IDEFICS study. Eur J Clin Nutr. 2015;69(7):811-816. doi:10.1038/ejen.2015.76
33. Al-Kadi A, Malik AM, Mansour AE. Rising incidence of obesity in Saudi residents. A threatening challenge for the surgeons. Int J Health Sci. 2018;12(1):45-49.

34. Zaccagni L, Barbieri D, Gualdi-Russo E. Body composition and physical activity in Italian university students. $J$ Transl Med. 2014;12:120-129. doi:10.1186/1479-5876-12-120

35. Ohlsson B, Manjer J. Sociodemographic and Lifestyle factors in relation to overweight defined by BMI and "Normal-Weight Obesity". J Obes. 2020;2020:2070297. doi:10.1155/2020/2070297

36. Männistö S, Harald K, Kontto J, et al. Dietary and lifestyle characteristics associated with normal-weight obesity: the National FINRISK 2007 Study. $\quad B r \quad J \quad$ Nutr. 2014;111(5):887-894. doi:10.1017/s0007114513002742

37. Amani R, Parohan M, Jomehzadeh N, Haghighizadeh MH. Dietary and biochemical characteristics associated with normal-weight obesity. Int J Vitam Nutr Res. 2019;89(5-6):331-336. doi:10.1024/ 0300-9831/a000477

38. Campos-Uscanga Y, Gutierrez-Ospina G, Morales-Romero J, RomoGonzalez T. Self-regulation of eating and physical activity is lower in obese female college students as compared to their normal weight counterparts. Eat Weight Disord-St. 2017;22(2):311-319. doi:10.1007/s40519-016-0338-9

39. Ogunbode AM, Ladipo MMA, Ajayi IO, Fatiregun AA. Obesity: an emerging disease. Niger $J$ Clin Pract. 2011;14(4):390-394. doi:10.4103/1119-3077.91741

40. Jung KI, Ock SM, Song CH. Weight change and mental health according to obesity in relatively healthy middle-aged Korean women: the Korea National Health and Nutrition Examination Survey 2014, 2016, 2018. J Affect Disord. 2021;287:174-181. doi:10.1016/j.jad.2021.02.017

41. Chalklin CG, Ryan Harper EG, Beamish AJ. Metabolic and Bariatric Surgery in Adolescents. Curr Obes Rep. 2021;10(2):61-69. doi:10.1007/s13679-021-00423-3

42. Sinha R, Jastreboff A. Stress as a common risk factor for obesity and addiction. Bio Psych. 2013;73(9):827-835. doi:10.1016/j. biopsych.2013.01.032

43. Koch FS, Sepa A, Ludvigsson J. Psychological Stress and obesity. J Pediatr-Us. 2008;153(6):839-844. doi:10.1016/j.jpeds.2008.06.016

44. Dutheil F, Chaplais E, Vilmant A, et al. Stress management in obesity during a thermal spa residential programme (ObesiStress): protocol for a randomised controlled trial study. BMJ Open. 2019;9(12): e027058. doi:10.1136/bmjopen-2018-027058

45. Czeglédi E. Options for stress management in obesity treatment. Orv Hetil. 2016;157(7):260-267. doi:10.1556/650.2016.30362

46. Robinson E, Hunger JM, Daly M. Perceived weight status and risk of weight gain across life in US and UK adults. Int $J$ Obes. 2015;39 (12):1721-1726. doi:10.1038/ijo.2015.143

47. Xenaki N, Bacopoulou F, Kokkinos A, Nicolaides NC, Chrousos GP, Darviri C. Impact of a stress management program on weight loss, mental health and lifestyle in adults with obesity: a randomized controlled trial. J Mol Biochem. 2018;7(2):78-84.

Diabetes, Metabolic Syndrome and Obesity: Targets and Therapy

\section{Dovepress}

\section{Publish your work in this journal}

Diabetes, Metabolic Syndrome and Obesity: Targets and Therapy is an international, peer-reviewed open-access journal committed to the rapid publication of the latest laboratory and clinical findings in the fields of diabetes, metabolic syndrome and obesity research. Original research, review, case reports, hypothesis formation, expert opinion and commentaries are all considered for publication. The manuscript management system is completely online and includes a very quick and fair peer-review system, which is all easy to use. Visit http://www.dovepress.com/testimonials.php to read real quotes from published authors.

Submit your manuscript here: https://www.dovepress.com/diabetes-metabolic-syndrome-and-obesity-targets-and-therapy-journal 